erwarten. Später ist infolge gelehrter erinnerung an die lat. bencnnung des Rheinlands, die vielleicht auch noch im volksmunde sich lebendig erhalten hatte, das lat. $p$ in dem Namen Ripuarii wiederhergestellt worden.

Als jüngere bildung ist Ripuarja 'Rheinland' nach dem muster von schon vorliegenden zusammensetzungen wie Chasuarja 'Haseland', Amsivarja 'Emsland' u. dgl. m. erst in der Römerzeit entstanden. Der gang der entwickelung mag also folgender gewesen sein: Von einem völkernamen wie Frisii wurde ein ländername germ. Frisiawja 'Friesenau' abgeleitet; von diesem lä̀ndernamen wurde dann (nur im römischen munde?) der völkername Frisiavi, Frisiavones gebildet. Ähnlich wird von flußnamen wie Amisis 'Ems' ein ländername germ. Amsivarja abgeleitet, woraus der volksname Amsivarii gebildet ist. Daß die festländischen stämme selbst die epichorischen namen gebrauchten, ist nicht erwiesen. Wenn den Angrivarii der lat. schriftsteller der alte name Angerii, die heutigen Engern entsprechen, so ist eher das gegenteil wahrscheinlich. In England und im norden sind freilich germ. völkernamen auf -varii beglaubigt. Wir kommen demnach zum schluß: der stammesname Ripuarii ist dem hybriden germ. ländernamen Ripuarja entnommen und bedeutete nach den vorstehenden ausführungen also ganz dasselbe wie unser heutiges 'Rheinländer'.

BERLIÑ.

SIGMUND FEIST.

\title{
ZUM HELIAND.
}

จ. $295 \mathrm{f}$.

Thô uuarð hugi Jôsepes, is môd giuuorrid, the im êr thea magar habda, usw.

So liest C, während M gidrôbid statt des unverständlichen giuuorrid bietet. Sinn und alliteration dürften gimerrid als ursprüngliche lesart ergeben. Das verbum ist im Heliand mehrfach belegt, vgl. besonders v. 5919: gimerrid unârun iro thes muodgithâhti, sowie aengl. môd gemyrred Jul. 412.

v. 2591. C: antthat mûdspelles megin obar man ferid M bietet hier mûd-spelles; 
v. $4358 \mathrm{f}$. : matspelli cumit

an thiustrea naht, al sô thiof ferid.

Hier haben beide hss. dieselbe form.

Vgl. über das vielbehandelte wort Braune, Ahd.leseb. ${ }^{7}$ s.190f: Beitr. 40,425 ff. 41, 192. Im ahd. findet sich nur der dat.mîspille Musp. v.57, im aisl. der nom. Múspell, der gen. Múspells, und der dat.-spelli. Abweichend von allen früheren erklärern erblicke ich in alts. mût-eine alte bezeichnung für 'feuchtigkeit, feuchte erde',

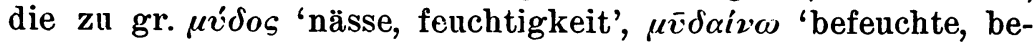
netze', $\mu \bar{v} \delta \alpha \lambda \varepsilon$ 'os 'feucht, naß' usw., lit. máudyti 'baden', skr. mudiv'a', air. muad 'wolke', lat. mustum 'most' usw. gehört. Im germ. ist die wurzel vertreten durch schwed.muta 'rieseln', mnd. mûten 'das gesicht waschen' (in Soest miutn), westf. müter 'kater' (Woeste), mhd. smuz 'schmutz' sowie nl. mooi 'schön', vgl. Boisacq s. $\mu v ́ \delta o s$, verf. K. Z. 28, 282 und 48, 156. - Das feuer des jüngsten tages verbrennt und zerstört die feuchte erde, vgl.

Musp. v. 52 f.:

muor varsunilhit sih;

ahâ artruknênt,

v. $58 \mathrm{f}$.: denne daz preita uuasal allaz varprinnit, enti vâir enti luft iz allaz arfurpit.

Mit mût dürfte dasselbe gemeint sein, was Musp. v.58 als uuasal bezeichnet wird. Die form mût- wäre demnach die ursprüngliche, während sich mûd- in M. v. 2591 leicht als 'umgekehrte schreibung' erklärt (auslautendes - $d$ wurde stimmlos, vgl. das häufige ant- und mein Alts. elementarbuch $§ 248$ ). Im ahd. würde man zunächst *mûzspelli erwarten, worin aber der schwund der spirans $z$ vor $s$ leicht begreiflich ist; im aisl. müßte ebenfalls $t$ vor $s$ geschwunden sein, wofür ich aus Noreen, Aisl. gram. 3 \$ 281, 2 fälle wie ansuar, anskote, anspiall, -styggr, $\operatorname{sun}(d)$ s und ebda. 10 solche wie hes $(t) s, k v i s(t) s$, fós $(t)$ syster als parallelen anführe.

Meine erklärung des dunklen wortes als 'feuchtigkeitszerstörer' dürfte also vom lautlichen wie vom semasiologischen standpunkte aus gleich unanfechtbar sein und sich den älteren deutungen getrost zur seite stellen.

v. 2619. the than êgan uuili alungan tir.

J)er zweite halbvers ist zu kurz. Wenn man nicht mit. (? afür aldarlangen schreiben will, wird man nach aengl. callınga oll allungan einsetzen miissen. 

v. :355:3 f. I. endi frágodun sân
firiunitlîcon
〈then) regrinblindun,
huuilic thar riki man
undiar themu folescepi furista uuâri.

V'gl. thein blindun v. 3580. Ourch then wird der sonst zu kur\%e ver's am einfachsten geheilt.

v. 3671. 'Thô nâhide neriendo Crist.

Der èrste halbver's ist zu kurz, aber leicht durch einfügung von selbo hinter nâhide zu heilen, vgl. dieselbe besserung in v. 5976: sitit imo thar $\langle s e l b o\rangle$, wo Behaghel meinen vorschlag angenommen hat. Als parallele möchte ich noch v. 2621 anführen: Sô gifragn ik that thô selto sunu drohtines.

\section{v. $4126 \mathrm{ff}$. an Hierusâlem, thar Iudeono uuas, heri handmahal endi hôbidstedi, grôt gumskepi grimmaro thioda.}

So druckt Behaghel - mir unverständlich. Die hs. C bietet allerdings so, aber M liest hereo endi handm., was sinnlos ist. Sievers schiebt nach $\mathbf{M}$ endi ein: heri endi handm., ebenso Heyne, und zwar ohne komma nach uuas. Sollte nicht heries handm. zu lesen sein, wobei heries den gen. Iudeono regieren würde? Das hereo von $M$ ließe auch an hêrost denken, doch will mir heries besser gefallen. Das komma nach uuas ist aber jedenfalls zu streichen!
v. $5798 \mathrm{ff}$. faran an fererhamon, that all thiu folda ansciann, thiu erða dunida, endi thia erlos uuroun an uuêkan hugie, uuardos Juðeono.

Das seltsame ansciann möchte ich nicht mit Kauffmann in arsciadh bessern (ein praefix $a r$ - gibt es im alts. nicht), sondern in ascall, also ein synonymon $\mathrm{zu}$ dunida. $n$ für $l$ findet sich auch in suncan = sulican M (vgl. mein Alts. elementarbuch $\S 177$ anm. 1), an- beruht auf praefixvertauschung, wenn nicht vorwegnahme des folgenden $n$ vorliegt, und das eingeschobene - $i$ - könnte durch ein praesens ${ }^{*}$ ascicllan mit palatalisiertem $c$ (vgl. Elementarb. $§ 242$ und nengl. chose(n) neben choose) erklärt werden.

v. $5061 \mathrm{ff}$.

Gengun im an uuarf samad

rinkos au rûna, bigunnun im râdan thô, huô sie geuuîsadin mid unârlôsun mannun mêngeuuitun an mahtigna Crist te giseggianne sundea thurh is selbes uuord 
Die bedeutung des unerklärten geuuîsadin muß nach dem zusammenhange 'fertig brächten' sein (vgl. Sievers zur stelle), und da ergibt sich als naheliegende besserung das mehrfach belegte genucrcadin. Die verschreibung erklärt sich leicht durch einfluß von giuuirlkan und giuûssian.

Ich benutze die gelegenheit, einige versehen im text und glossar von Behaghels neuester (3.) ausgabe des Heliand zu bessern: v. 144 l. tuêntig, 215 l. thiu, 490 l. te, 549 l. slî̀uurdean, 822 l. sorga, 990 l. endi, 1214 l. lêf, 1305 l. mâdmundic, 1310 l. bicnêgan, s. 58 gehören die drei ersten zeilen der fußnote $\mathrm{zu}$ s. 57, v. 1972 l. hêriu, 2028 l. gitrîoda, 4089 fehlt das zeichen des redeschlusses nach godes, 4203 l. pâscha (westf. $p \bar{\emptyset} s k$ ), 4320 l. endi heri lêdid, 4517 l. gôdo, 4568 l. quale, 5061 u. 5071 l. uuarf, 5379 l. quala, 5409 u. 5413 l. hêri. Bei den eigennamen fehlen häufig die längezeichen, auch wo das metrum sie fordert.

KIEL.

F. HOLTHAUSEN.

\section{DIE ALTDEU'TSCHEN ADVERBIEN VON HOCH.}

In den Göttingischen gelehrten nachrichten 1918, s. 378 behandelt Edw. Schröder 'das adverbium zu hôch'. Er legt in umfassender weise das vorkommen des adverbs hô dar und sichert für das mhd. auch die form hôch für das adverb. In durchaus zutreffender weise widerlegt er die bisherigen erklärungen der form $h \hat{o}$; er selbst leitet sie unmittelbar aus der form hôch ab mit abfall des schließenden $c h$; die form hôch ist ihm ein adverbialer accusativ.

Diese erklärung der erscheinungen ruft starke bedenken hervor. Ich wende mich zunächst zu der form hô. Es wäre schon auffallend, wenn die weit verbreitete form ho entstanden wäre aus der form hôch, die nur in einigen wenigen mhd. stellen gefunden wird. Zweitens: daß ch abgefallen sei, läßt sich nur für solche gebiete annehmen, in denen auch andere auslautende $c h$ untergegangen sind; einen solchen machweis zu führen, hat Schröder nicht versucht, und er würde auch schwerlich gelingen. Drittens und vor allem: schroiders 